\title{
Resistance to 1 hydroxyvitamin D? A challenging case
}

\author{
Department of Diabetes and Endocrinology, West Middleses Hospital, London
}

Introduction:

Hypoparathyroidism following parathyroidectomy is commonly treated with activated vitamin D, (alfacalcidol and calcitriol).

Alfacalcidol (1- hydroxyvitamin $\mathrm{D}$ ) is converted by hepatic 25 -hydroxylase to generate calcitriol (1,25-dihydroxyvitamin $\mathrm{D})$, to act on target cells. ${ }^{1}$

Case Presentation:

We present the case of 66 year old man who was admitted with chest pain in November 2016 and found to have corrected calcium of $1.5 \mathrm{mmol} /$ litre and PO4 $2.7 \mathrm{mmol} /$ litre. He had a background of CKD 3, CVA, CCF, IHD, hypoparathyroidism (following parathyroidectomy in 1987) and had been on alfacalcidol

$1 \mathrm{mcg}$ once daily since then. He had a prolonged admission from April 2016 to August 2016 following fall and on discharge, his alfacalcidol was stopped (for no apparent reason). Following readmission, he was given intravenous calcium and restarted on oral calcium supplements and alfacalcidol. Despite this, his hypocalcaemia failed to improve regardless of his calcium tablets \& alfacalcidol being increased to $1500 \mathrm{mg}$ TDS and $2.5 \mathrm{mcg}$ OD respectively. His liver function tests were normal and CT abdomen did not show any hepatic abnormality. Following this, (in the event of malabsorption) his alfacalcidol was given intravenously but with no improvement, so this was converted to calcitriol $1 \mathrm{mcg}$ OD with correction of his serum calcium within 48 hours.

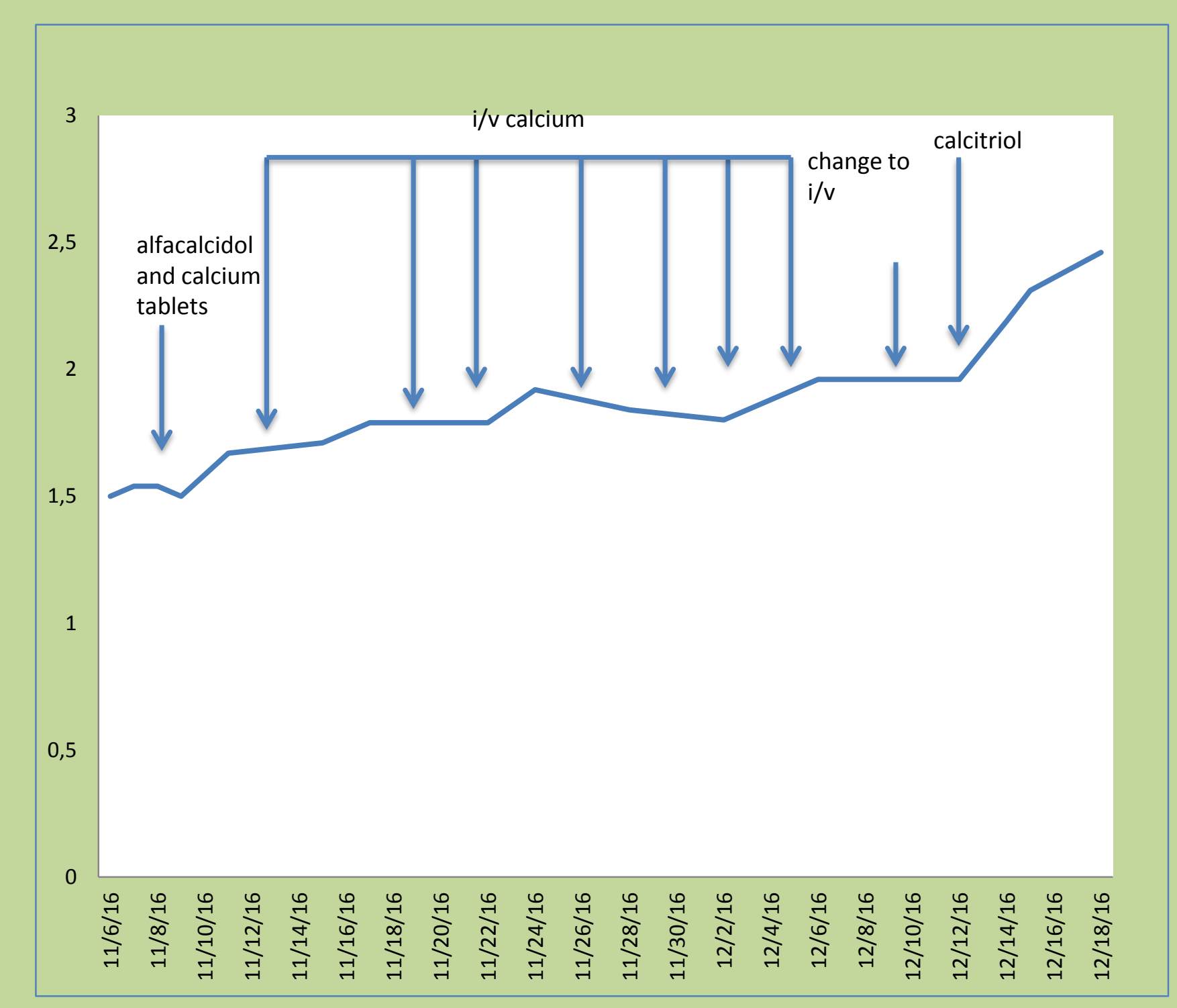

- Conclusion:

We advocate that 25 hydroxylation can be impaired without radiological or biochemical markers of liver abnormality. Our case highlights that patients who originally respond to 1 hydroxyvitamin $D$ may develop resistance after a period of treatment, which can be alleviated with use of 1,25 hydroxyvitamin D instead.

- Reference:

1. . KRAIWIPORN KIATTISUNTHORN, KITTIKARN WUTYAM, ARTIT INDRANOI and SOMKIAT VASUVATTAKUL (2010) Randamized trial comparing pulse calcitriol and alfacalcidol for the treatment of secondary hyperparathyroidism in haemodialysis patients. Nephrology 16 (2011) 277-284 